\title{
Contents, Vol. 112, 1946
}

\section{Index}

Ammann, E., Zur Vererbung der Henieralopia hereditaria

und tapeto-retinalen Degeneration 78

Bíschler, V., vide Streiff, E. B.

Doesschate, J. Ten, Visual Acuity and Distribution of Percipient Elements on the Retina 1

Ferrié, J., Lesions maculaires d'aspect dégénératif et tuber-

culose. (Avec planche I.) 135

- et Grynfeltt, J., Etude anatomo-clinique de certaines formes de la Maladie de Besnier-Boeck-

Schaumann. Essai de pathogénèse. (Avec planche II.) . . . . 193

Garcia-Miranda, A., «Umgekehrtes» Foster-Kennedy-Syn-

drom 72

Gardileié, A., Ein Beitrag zur Extraktion der am Bodendes Glaskörpers liegenden Linse mit

Hochfrequenz-nadel nach Jeß-L-acarrère 255

Gát, L., und Orbán, L., Nach Staroperation auftretende De-

lirien 335

Gaus, J., Die Tiefenwahrniehmung im indirekten Sehen . 267

Goldmann, H., Weitere Mitteilung über den Abfluß des

Kammerwassers beim Menschen. (Hierzu Tafel III.) . 344

Gruber, M., «Cornea verticillata». 2. Mitteilung ... 88

Grynfeltt, J., vide Ferrié, J.

Gsell, ()., Rehsteiner, K., und Verrey, F., Iridocyclitis alsSpätfolge von Leptospirosis Pomona

(Schweinehüter-krankheit). Agglutinin- und Lymphozytose-Befund imKammerwasser 320

Guiral, J., vide Jean-Sédan.

Haefeli, W., Die Fluorescein-Permeabilität der Blut-Kam-

merwasser-Scliranke des gesunden Auges . . . 226

Henkes, H. E., On the Distribution of Glutathione and Vitamin C in the Lens and Cornea 113

Hermann, C, La dystrophie grillagée de la cornée. Contribution clinique et anatomo-

pathologique.) (Avecplanche IV.) 350

Jabïonski, W., Augenärztliche Eindrücke im Orient . .

Jean-Sédan, Guiral, J., et Reínhard, Ch.-H., Kyste sébacé orbitaire, coïncidant avec une volumineuse lacune du squelette cranio-facial et prètant à confusion avec une méningocèle, voire une encéphalo-méningocèle . . 57

Landau, E., Contribution à ГHistoIogie de Гæil (II) . . 129

Loon, J. A. van, The Cold Pressor Test in Ophthalmology . 63

Mulock Houwer, A. W., Amblyopia cum Polyneuropathia

Caused by Starvation 177

- Potassiumiodide and Vitreous Opacities

Orbán, $L \cdot$., vide Gát, L. 
Payot, P., Über die Beeinflussung der Histaminchemosis des Kaninchens durch Anaesthetica 25

Poleff, L., A propos de la classification des tumeurs épithé-

liales cornéennes 287

Rehsteiner, K., vide Gsell, O.

Reinhard, F., vide Jean-Sédan.

Roussel, F., vide Weekers, R.

Streiff, E. B., et Bischler, V., La precision de la méthode

ophtalmodynamométrique 19

Veelen, A. W. C, van, Doppelseitige spontane Descemet-

Ruptur 149

Verrey, F., vide Gsell, 0.

Weekers, R., et Roussel, F., Introduction a Гétude de la frequence de fusion en clínique 305

GESELLSCHAFTSBERICHTE - SOCIETY TRANSACTIONS - SOCIÉTÉS

Johannes 1seli zum 70. Geburtstae

Groupe ophtalmologique du L·éman, seance des 10 et 11 mars 194õ à

Geneve $\quad 47$

Vereinigung der Basler Augenärzte. Basel, den 12. November 1945 . . 92Report of the 109th

Meeting of the Netherlands Ophthalniological Societyat the Netherlands Ophthalmic Hospital at

Utrecht. On Saturday, January 12th aikl Sunday January 13th, 1946

RELATA

Heßberg, R.J., Status of Compensation for Ocular Injuries in the United

Slates, by Morris Davidson 110

- Review of Contributions of World War II to Ophthalmology . 292

B. BUCHBESPRECHUNGEN - BOOKS REVIEW - LIVRES NOUVEAUX

Ill

301

363 